\title{
Detection of gear defects using method of wavelet decomposition and cross-correlation - Experi- ment to develop a feasible detection method
}

\author{
R. Lullus Lambang $G$ Hidayat $^{1,}{ }^{*}$, and Budi Santoso ${ }^{1}$ \\ ${ }^{1}$ Mechanical Engineering Dep. Faculty of Engineering Universitas Sebelas Maret Indonesia
}

\begin{abstract}
Detection of machine component failure is very important to be properly applied in a maintenance program in industries. The objective of this research is to detect gear fault using wavelet transforms. The vibration signal is acquired with accelerometer mounted at bearing houses of 2 parallel shafts with 2 spur gears (28 tooth). The gears are rotated at 1200 RPM and the spectrum is displayed. The spectrum cannot indicate gear mesh frequencies (GMF), because they are covered with frequencies such as natural and harmonic frequencies of rotating shaft. This research have developed a method to obtain GMF using wavelet decomposition and cross correlation. The results showed that with FFT applied to cross-correlation of wavelet detil components,, spectrum of visible and distinctable GMF has been obtained.
\end{abstract}

\section{Background}

Gear units are parts of machines serve to transmit speed and torque between two shafts. The units are used in wind turbine [1], automotive and helicopter engines [2]. During using, the gear defects like wear, broken, cracked usually occure. The damage should be detected in a maintenance program. Gear condition monitoring is therefore required in order to improve safety and reduce operating costs.

In this research, a rig consists of 2 shafts with 2 gear is constructed . One gear is normal gear while the other has a partially/full toothcut. Electric motor is used to rotate one shaft to 1200 RPM. Vibration signal is then obtained using accelerometer mounted on the bearing house. The signal processing method uses only FFT has disadvantages, i.e. the frequency associated with fault condition must be known first and if the signal has a low signal to noise ratio or there are many other vibration components then peaks correspond to failure frequency cannot be clearly indicated $[3,4]$.

This research uses wavelet transformation to indicate GMF features so that gear failure i.e. full / partial toothcut gear can be detected. Wavelet-based signal processing for gear fault detection had been investigated by [4] and [5]. Further studies of wavelet transform applications on vibration signals for gear fault detection have been also performed by [6] and [7] that discuss of wavelet decomposition and feature extraction using PCA.

*lulus_1@staff.uns.ac.id; lulus_1@yahoo.com 


\section{Theoretical Basis}

Gears are used in almost transmission systems, power systems and automotive. The gears have to rotate at a constant speed [4]. If the gear rotates at an unstable speed then one of the gears will spin fast and vibrations as an indicative of damage is produced [2]. Figure 1(a) shows the structure of a spur gear and the terms that are often used in discussions.

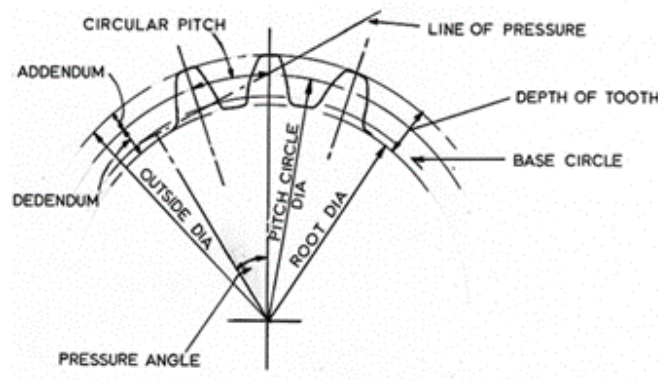

(a)

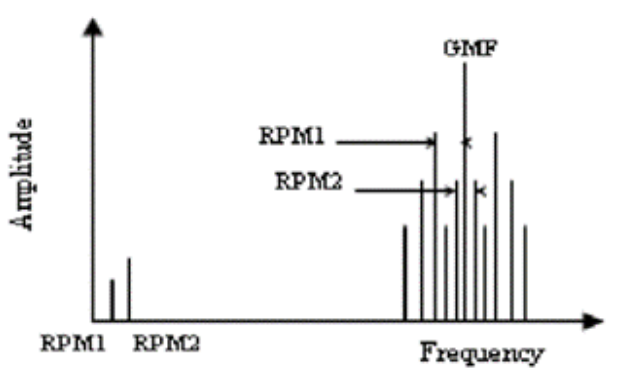

(b)

Fig. 1. Spur gear definition and corresponding failure spectrum

\subsection{Gear mesh frequencies}

The main source of vibration and noise gear is the dynamic excitation of the varying stiffness of the meshed teethgears. The mesh stiffness or elastic bending tooth changes as much as the changes in the number of contacts. As the gear rotates, two teeth meet at a pitch point. GMF is therefore defined as the number of gear teeth multiplied by its rotational speed. GMF and sideband frequencies are shown in Fig. 1(b).

\subsection{Wavelet Transformation- Multiresolution Analysis}

The wavelet transform of a signal is an interesting and relatively new tool applied for vibration analysis. The basic idea of wavelets is to analyze signals based on scale and dilatation parameters. Multiresolution analysis involves the analysis, processing and synthesis that breaks down a signal into coefficients of the scaling and wavelet functions in different resolution levels. [8].

Wavelet function : Suppose a signal $x=x(t)$ is expressed as a 2-dimensional function, $\psi_{j, k}$ (t), as follows:

Wavelet function is defined as:

$$
x(t)=\sum_{j} \sum_{k} c_{j, k} \psi_{j, k}(t)
$$

$$
\psi_{j, k}(t)=C \cdot \psi\left(2^{j} t-k\right)
$$

where $C$ is a constant, $j$ is dilation parameter and $k$ is shift parameter. Equation (1) can be rewritten as a summation of functions, that is :

$$
x(t)=\sum_{i=-\infty}^{j} \sum_{k} c_{i, k} \psi_{i, k}(t)=\sum_{i=-\infty}^{j-1} \sum_{k} c_{i, k} \psi_{i, k}(t)+\sum_{k} c_{j, k} \psi_{j, k}(t)
$$

Scaling function : Given a scaling function $\phi_{j, m}(t)$, then the analysis of signal $x(t)$ is $c A_{0}(m)=\int_{-\infty}^{\infty} x(t) \phi_{j, m}(t) d t$. The synthesis of signal $x(t)$ uses the coefficient $c A_{0}(m)$ yields an approximation of $x(t)$, that is written as follows:

$$
x(t) \approx \sum_{m} c A_{0}(m) \phi_{j, m}(t)
$$


Besides of the approximation of $x(t)$ in eq. (4), the signal $x(t)$ can be expressed as:

$$
x(t)=\sum_{k} c A_{0}(k) \phi_{j, k}(t)=\sum_{k} c A_{1}(k) \phi_{j-1, k}(t)+\sum_{k} c D_{1}(k) \psi_{j-1, k}(t)
$$

The approximation coefficient, $c A_{l}(k)$ and the detail coefficients, $c D_{l}(k)$, are determined as $c A_{1(k)}=\left\langle x(t), \phi_{j-1, k}(t)\right\rangle$ dan $c D_{1}(k)=\left\langle x(t), \psi_{j-1, k}(t)\right\rangle$, respectively. Eq. (5) is rewritten as:

$$
x(t)=\sum_{k} c A_{1}(k) \phi_{j-1, k}(t)+\sum_{k} c D_{1}(k) \psi_{j-1, k}(t)=A_{1}(t)+D_{1}(t)
$$

Component $A_{l}(t)$ can be decomposed to the next levels to obtain:

$$
\begin{aligned}
x(t) & =A_{2}(t)+D_{2}(t)+D_{1}(t) \\
& =A_{3}(t)+D_{3}(t)+D_{2}(t)+D_{1}(t) \\
& =A_{4}(t)+D_{4}(t)+D_{3}(t)+D_{2}(t)+D_{1}(t)
\end{aligned}
$$

Eq. (7) implies that the signal $x(t)$ can be decomposed into components of lower level, i.e. as a scaling function, $\phi_{j-1, k}(t)$ and wavelet function, $\psi_{j-1, k}(t)$. Signal decomposition is then proceed up to a desired level.

Signal decomposition can be performed using Mallat algorithm. A a low-pass filter (LPF) $h(k)$ and a high-pass filter (HPF), $g(k)$ are employed. The filters are defined as $g(k)=$ $(-1)^{k} h(1-k)$, called Quadrature Mirror Filters (QMF). The wavelet function and scaling function use LPF and HPF are $\phi(t)=\sqrt{2} \sum_{k} h(k) \phi(2 t-k)$ and $\psi(t)=\sqrt{2} \sum_{k} g(k) \psi(2 t-k)$, respectively. Furthermore, the approximation coefficient $c A_{l}$ and the detail coefficient $c D_{l}$ are determined as $c A_{1}=\sum_{n} h(n-2 k) c A_{0}(n)$ and $c D_{1}=\sum_{n} g(n-2 k) c A_{0}(n)$, respectively. The approximate and detailed signals therefore are $A_{1}(t)=\sum_{k} c A_{1}(k) \phi_{j-1, k}(t)$ and $D_{1}(t)=$ $\sum_{k} c D_{1}(k) \psi_{j-1, k}(t)$, respectively.

The signal analysis using wavelet decomposition includes selection of wavelet function, scaling function, lowpass and highpass filters. These determine the ability of wavelet decomposition to capture transient properties in a signal. This research uses $d b 4$ wavelet that is suitable for vibration signals analysis $[7,12]$.

\subsection{The signal energy of the wavelet detail component}

Parseval theorem states that the signal energy is expressed as the sum of the energy of components of the signal. This also applies to wavelet decomposition as:

$$
\sum_{m=0}^{M-1}|x(t)|^{2}=\sum_{m=0}^{M-1}\left|c A_{L}(m)\right|^{2}+\sum_{l=1}^{L} \sum_{m=0}^{(M-1) / l}\left|c D_{L}(m)\right|^{2}
$$

where $t$ is the discrete time, $l$ is the $l$-th decomposition level, $m=m$-th sample data, $L$ is the number of decomposition level and $M$ is the number of samples. The signal energy of the $L$ level detail component, $c D_{L}$ is the dot product of the wavelet detail coefficients, $\sum_{m=0}^{(M-1) / l}\left|c D_{L}(m)\right|^{2}$. Then $D e_{\max }$ is determined as the wavelet detail components with the highest signal energy among $1,2, \ldots L$ level and $D e_{\max -1}$ is determined as wavelet detail components with signal energy right to lower than detail component energy, $D e_{\max }$.

\subsection{Cross correlation of wavelet coefficient}

The cross correlation of the wavelet decomposition coefficients is calculated as :

$$
\left\langle D_{e_{\max }} * D_{e_{\text {max }}-1}\right\rangle[n]=\frac{1}{M} \sum_{m=1}^{M} D_{e_{-} \max }(m) D_{e_{-} \max -1}(n+m)
$$

where $n$ is the $n$-th component of the cross correlation. Cross correlation provides information of similarity between 2 signals. This operation is also used to increase signal periodicity. Cross correlation of wavelet coefficients for vibration signals analysis had been performed in the research of $[12,13,14]$. 


\section{Research method}

The research was conducted at Laboratory of Vibration and Maintenance of Mechanical Engineering Dept, Faculty of Engineering, Sebelas Maret University. The schematic of rig used in this research is shown in Fig. 2. The figure shows one shaft is connected to motor using a flexible coupling. The driver gear and driven gear are spur gears with 28 tooth. Driver gear is of normal gear whereas driven gear with $25 \%, 50 \% 75 \%$ and $100 \%$ toothcut are used.
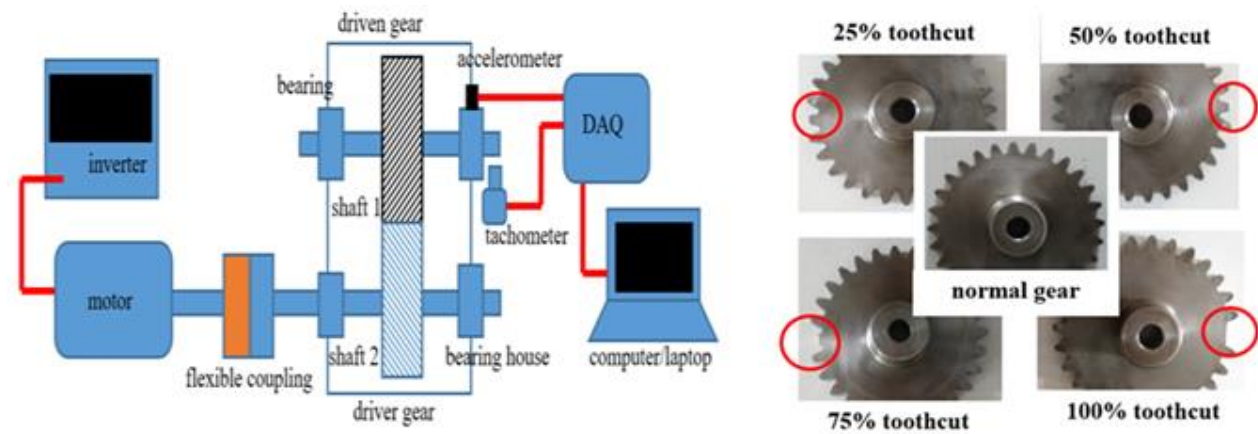

Fig. 2. Schematic of gears rig and driven gears

\subsection{Research Implementation}

The implementation include the set up of the gear rig and the installation of sensors. The data acquiring steps consists of (1) turning on an electric motor and set it to a constant speed of 1200 rpm ; (2) record vibration signal from accelerometer using DAQ; (3) measure shaft rotation using infrared tachometer, and (4) use computer to store vibration data. The signal data from Dewesoft ${ }^{\circledR}$ DAQ is converted to (.mat) files to be read by MATLAB. The signal is decomposed and the energy of wavelet detil coefficients sets are calculated. The set with largest energy is the most represents the gear defect. This decomposition method has been developed previously for unbalance and misallignment detections $[11,12]$.

\section{Research method}

The fault detection is generally performed by observing a particular pattern on the spectrum of recorded signal. The failure pattern is indicated as peaks at frequency of an integer multiplied by 1 X RPM. The proposed method uses wavelet decomposition to obtain transient properties of a signal. Wavelet decomposition have a drawback of low time resolution at high frequency because the frequency resolution multiplied by the time resolution is constant. Because of this, cross correlation is used to improve the performance of wavelet decomposition at high frequencies. Futhermore a spectrum to indicate GMF is constructed.

\subsection{Vibration Signals and Wavelet Decomposition Components}

Signals of normal, 50\% and 75\% toothcut gears are shown in Figs. 3(a) and (c). Signals of toothcut gears contains more peaks than the one of normal gear, especially for $75 \%$ toothcut gear. This is because impact between defect gear teeth and normal gear teeth is occured more rather than friction contact between the defect teeth with the normal teeth. The wavelet detail coefficients of the signal of toothcut gear also has a magnitude larger than the normal gear, compared using the same scale as shown in Figs. 3(b) and (d). 

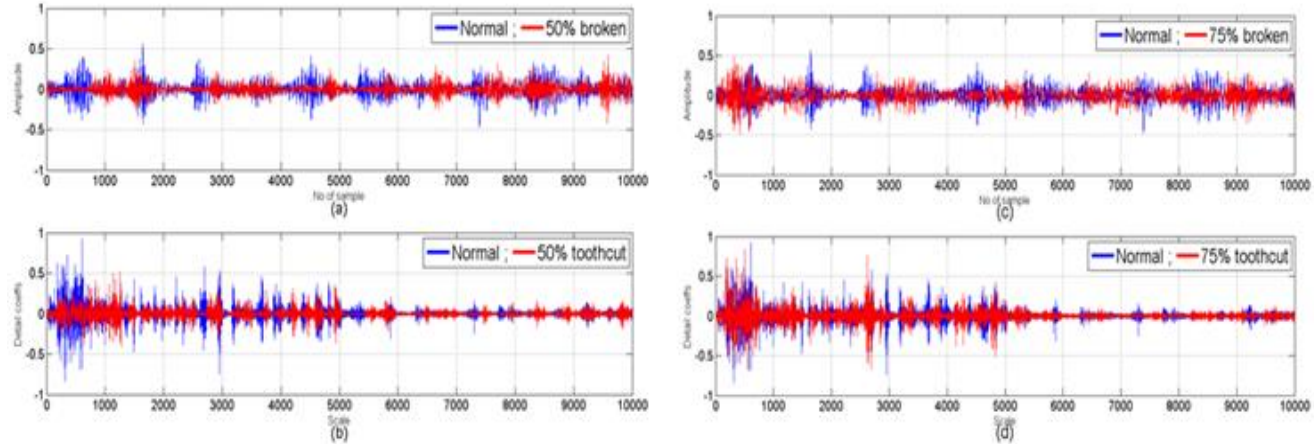

Fig. 3. Gear signal : 50\% toothcut (a) time domain; (b) detail coefficients; $75 \%$ toothcut (c) time domain; (d) detail coefficients.

\subsection{Spectrum of $25 \%$ and $100 \%$ tooth-cut gears}

The spectrum of normal gear, $25 \%$ and $100 \%$ toothcut gears are shown in Figs 4(a) and (c), respectively. The spectrum cannot indicate amplitudes of the signals of $25 \%$ toothcut gear larger than the normal gear signals. The normal and defect gears also cannot be distinguished in time domain signals. The proposed signal processing uses wavelet decomposition-cross correlationpower spectral show fairly different spectrum of normal and $25 \%$ toothcut gears as shown in Fig. 4(b). GMF is indicated at $551 \mathrm{~Hz}(\mathrm{GMF}$ is equal $28 \times(1200 / 60)=560 \mathrm{~Hz})$ as peak of 0.0081 .
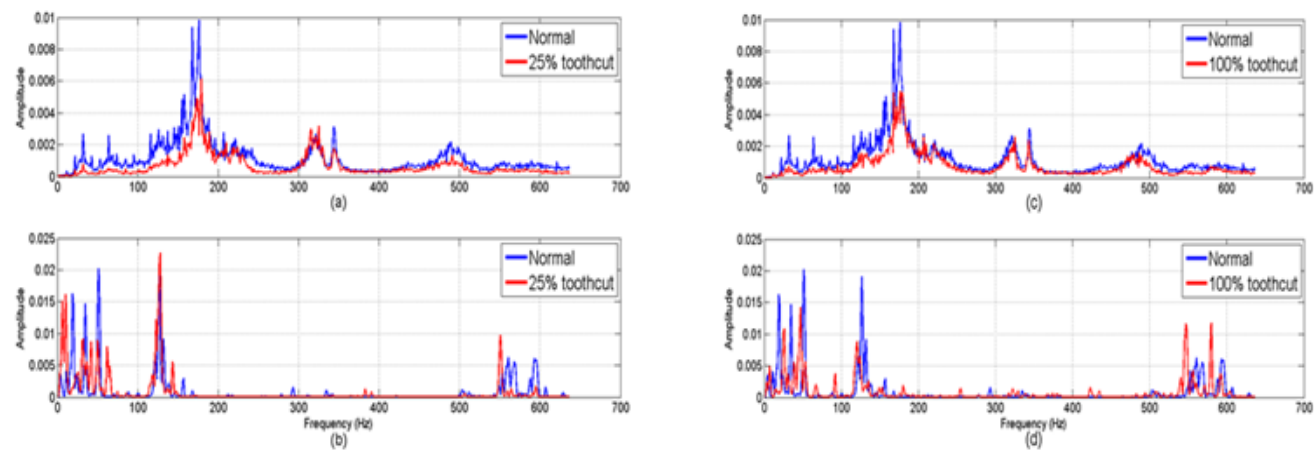

Fig. 4. Gear spectrum: 25\% toothcut (a) FFT; (b) proposed method; $100 \%$ toothcut (c) FFT; (d) proposed method

Figure 4(c) shows the spectrum of $100 \%$ toothcut gear signal with almost all peaks lower than the peaks of the normal gear signals. The missing part of $100 \%$ toothcut gear reduces the number of impacts between teeth gear mating. This is indicated by small amplitudes for medium-high scale, compared with normal gear signals in time domain signal and wavelet detail components (not showed). Figure 4(c) does not show GMF while Figure 4(d) shows clearly GMF with peak of 0.0111 at $551 \mathrm{~Hz}$ and sidebands with peak of 0.010 and 0.005 at 546 and $568 \mathrm{~Hz}$, respectively.

\subsection{Performance of the proposed method}

The performance of proposed method is evaluated by comparing to the FFT used to display the gear failure features as shown in Table 1 . 
Table 1. The features in the spectrum obtained by FFT and proposed method (PM)

\begin{tabular}{|c|c|c|c|c|c|}
\hline \multicolumn{3}{|c|}{$25 \%$ toothcut gear } & \multicolumn{3}{|c|}{$50 \%$ toothcut gear } \\
\hline Feature & FFT & PM & Feature & FFT & PM \\
\hline GMF & NE & $\mathrm{E}$ & GMF & $\mathrm{NE}$ & NCE \\
\hline Side band frequencies & $\mathrm{NE}$ & $\mathrm{NCE}$ & Side band frequencies & $\mathrm{NE}$ & $\mathrm{NE}$ \\
\hline 1X RPM; 2X RPM & $\mathrm{E} ; \mathrm{NCE}$ & E; E & 1X RPM; 2X RPM & E;NCE & $\mathrm{E} ; \mathrm{NE}$ \\
\hline Harmonics freqs of RPM & $\mathrm{NE}$ & E (6XRPM) & Harmonics freqs. of RPM & $\mathrm{NE}$ & E (6 XRPM) \\
\hline \multicolumn{3}{|c|}{$75 \%$ toothcut gear } & \multicolumn{3}{|c|}{$100 \%$ toothcut gear } \\
\hline Feature & FFT & PM & Feature & FFT & PM \\
\hline GMF & NCE & E & GMF & $\mathrm{NE}$ & $\mathrm{E}$ \\
\hline Side band frequencies & NCE & $\mathrm{E}$ & Side band frequencies & $\mathrm{NE}$ & $\mathrm{E}$ \\
\hline 1X RPM; 2X RPM & E; NCE & E; E & 1X RPM; 2X RPM & NCE;E & $\mathrm{E} ; \mathrm{E}$ \\
\hline Harmonics freqs of RPM & $\mathrm{E}(9 \mathrm{X})$ & $\mathrm{E}$ (6XRPM) & Harmonics freqs. of RPM & $\mathrm{E}(9 \mathrm{X})$ & $\mathrm{E}_{(6 \mathrm{XRPM})}$ \\
\hline Harmonics near GMF & NCE & $\mathrm{E}$ & Harmonics near GMF & NCE & $\mathrm{E}$ \\
\hline
\end{tabular}

$\mathrm{E}=$ excited, $\mathrm{NE}=$ not excited, $\mathrm{NCE}=$ Not clearly excited

\section{Conclusion}

This research has developed a new method for gear failure detection using method of wavelet decomposition-cross correlation-power spectra. The spectrum that shows distinct peaks on GMF and sideband frequencies as an indication of the gear damage is successfuly obtained. The $\mathrm{db} 4$ wavelet function for the vibration signal was used. It is highly recommended to use another wavelet function that has the same characteristics as gear fault for the next researches.

\section{References}

1. W. Wei, M. Xiaoping, W. Qianjin, Int. J. Control Autom. 8. No. 11, pp. 127-134 http:/dx.doi.org/10.14257/ijca.2015.8.11.13 (2015)

2. Y. Qu, D. He, J. Yoon, B. Van Hecke, E. Bechhoefer, and J. Zhu, Sensors, 14, 1372-1393; doi:10.3390/s140101372 (2014)

3. M. H. Lim, and MS. Leong, AIME, Hindawi Publishing Corporation, 2, Article ID 625863 (2013).

4. P. Gupta, S. B. Wadkar, AVE, 7(2), pp 181-195, (2008)

5. J. Chebil a, G. Noelb , M. Mesbah, M. Deriche, JJMIE 3, No. 4, ISSN 1995-6665 Pages 260 - 267, December (2009)

6. M. Ayad, Dj. Chikouche, N. Boukezzoula, M. Rezki, Int. Conference on Control, Engineering \& Information Technology (CEIT'14) Proceedings - Copyright IPCO-2014 ISSN 2356-5608

7. H. Bendjama, S. Boucherit, S., JTAM, 54.2 pp 659-670, Warsaw, (2016)

8. Z. Hong-tu, Y. Jing, Proceeding of the International Symposium on Electronic Commerce (2010)

9. H. Bendjama, S. Bouhouche, and S. Boucherit, IJMLC, Vol.2 No. 1 February 2012

10. D. Kanneg, W. Wang, JSIP, 2, 322-329. doi:10.4236/jsip.2011.24046 Published Online November (2011) 